Bundesgesundheitsbl 2009 $52: 887-888$

DOI 10.1007/s00103-009-0962-x

(c) Springer-Verlag 2009

B.M. Kurth · Robert Koch-Institut, Berlin

\title{
Über Ziele \\ und deren Erreichung
}

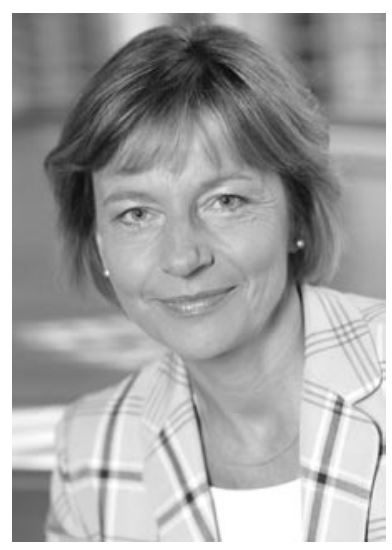

Aus dem etwas „verstaubten Amtsblättchen", das bis 1998 vornehmlich amtliche Mitteilungen und Bekanntmachungen aus den Herausgeberinstitutionen publizierte und darüber hinaus den Ruf genoss, für Publikationen "die sonst keiner nimmt", die letzte Anlaufstelle zu sein, ist ein attraktives Journal geworden. Seit Juli 2004 ist es in Medline gelistet, seit August 2007 zusätzlich noch in Scopus (der ElsevierDatenbank). Seit Oktober 2008 ist es zudem im Science Citation Index Expanded (SCIE) indexiert, der die Grundlage für die Ermittlung der Impact-Faktoren bildet, die im Journal Citation Report/Science Edition publiziert werden. Die SCIE-Indexierung heißt, dass das Blatt nun einer Impactfaktorberechnung unterliegt.

Das Themenspektrum des Bundesgesundheitsblattes spannt einen weiten Bogen, was durch eine zufällige Auswahl von Heften belegt sein soll:

- Heft 4/2001 Schwerpunktthema: BSE,

- Heft 3/2002 Schwerpunktthema:

Qualitätssicherung im Gesundheitswesen,

- Heft 8/2003 Schwerpunktthema: Gesundheitsökonomie und Versorgungsforschung,
- Heft 4/2004 Schwerpunktthema: Krankenhaushygiene,

- Heft 10/2005 Schwerpunktthema: ÖGD und Public Health,

- Heft 6/2006 Schwerpunktthema: Alter(n) und Gesundheit,

- Doppelheft 5 und 6/2007 Schwerpunktthema: Ergebnisse des Kinderund Jugendgesundheitssurveys,

- Heft 4/20o8 Schwerpunktthema: Depression,

- Heft 8 und Heft 9/2009 Schwerpunktthema: Universitätsmedizin im Wandel.

All diese Themenschwerpunkte werden dem formulierten Anspruch gerecht, „Fragestellungen und Bereiche zu reflektieren, mit denen sich das öffentliche Gesundheitswesen und die staatliche Gesundheitspolitik auseinandersetzen".

Das Bundesgesundheitsblatt hat in den letzten zehn Jahren eine erfolgreiche Entwicklung genommen, was den Autoren, dem Herausgeberbeirat und nicht zuletzt der seit 2001 zuständigen Redakteurin Frau Dr. Heidemarie Rohdewohld zu danken ist.

Das aktuell vorliegende Heft, das keinem Schwerpunktthema folgt, sondern ein sogenanntes "Freies Heft" ist, demonstriert auf eigene und überzeugende Art und Weise, die anspruchsvolle langfristig orientierte Ausrichtung der Zeitschrift: Drei Veröffentlichungen befassen sich mit der Evaluation von Gesundheitszielen. Diese Publikationen sind eine folgerichtige Fortsetzung einer Publikationsreihe zu Gesundheitszielen, die 2003 angefangen wurde. Beginnend mit Heft 2/2003, in dem die nationalen Gesundheitsziele für die Bundesrepublik 
Deutschland und die Kriterien ihrer Auswahl komplex dargestellt wurden, setzte sich die Publikationsreihe zu den Gesundheitszielen fort. Zum Beispiel im November 2006 durch einen Beitrag von Frau Prof. Maschewsky-Schneider et al. zur Evaluation des Gesundheitsziels „Tabakkonsum reduzieren" und im Juli 2009 zu den Auswahlund Evaluationskriterien für das neue Gesundheitsziel „Gesund altern". Die drei aktuell vorgelegten Arbeiten befassen sich mit den Evaluationskriterien für die Gesundheitsziele „Gesundheitliche Kompetenz erhöhen Patientinnensouveränität stärken" (K. Horch et al.) sowie „Depressive Erkrankung: Verhindern, Früherkennen, nachhaltig behandeln" (I. Bermejo et al). Der Beitrag von T. Lampert et al. beleuchtet die Entwicklung und Evaluation der nationalen Gesundheitsziele für Kinder und Jugendliche und fragt, welchen Beitrag der Kinder- und Jugendgesundheitssurvey des Robert Koch-Instituts hier leistet. Alle drei Arbeiten machen deutlich, dass die Auswahl und Formulierung von Zielen nicht den Endpunkt der Gesundheitszielebewegung darstellen. Vielmehr ist es erforderlich, sich über die Wege zum Ziel zu verständigen, Zwischenetappen und Kriterien für Ergebnisevaluationen festzulegen und die Weiterentwicklung der Ziele zu planen.

Ebenso wenig kann das Bundesgesundheitsblatt nunmehr für sich und alle Zeiten davon ausgehen, die vor zehn Jahren formulierten Ziele erreicht zu haben. Erreichtes muss bewahrt und weiterentwickelt werden. Evaluation ist ein Prozess. In diesem Sinne: Auf die nächsten Publikationen $z u$ den Ergebnissen des Gesundheitszieleprozesses und auf die nächsten zehn Jahre Bundesgesundheitsblatt.

Ihre

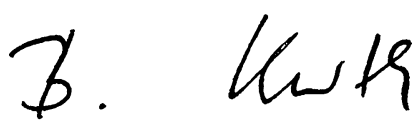

Bärbel-Maria Kurth

\section{Korrespondenzadresse \\ Dr. Bärbel-Maria Kurth}

Robert Koch-Institut,

Postfach 650261,

13302 Berlin

\section{W. Schwenk, C. Spies, J. M. Müller (Hrsg.) Fast Track in der operativen Medizin}

Heidelberg: Springer Medizin Verlag 2009, 1. Auflage, 786 S., (ISBN 978-3-540-39708-3), gebunden, 89.95 EUR

Der Begriff des "Fast Track" hat sich in den letzten 10 Jahren in der Chirurgie fest etabliert. Anfänglich noch mit Skepsis und Zweifel begegnet ist er zum Inbegriff eines modernen perioperativen Managements chirurgischer Patienten geworden; maßgeblich getragen durch die interdisziplinäre Zusammenarbeit von Ärzten der operativen Disziplinen, Anästhesisten sowie Pflegepersonal und Physiotherapeuten. Dabei steht nicht (wie der Begriff suggerieren mag) der zeitliche Gewinn sondern vielmehr die komplikationsarme, möglichst physiologische Rehabilitation des gut informierten Patienten unter Einschluss wissenschaftlicher Evidenz im Vordergrund.

Mit "Fast Track in der operativen Medizin" liegt nun das erste deutschsprachige, umfangreiche Werk zu dieser Thematik, geschrieben von Kollegen der Anästhesie, Chirurgie, Gynäkologie, Urologie und der Pflege, vor. Das Buch ist in einen allgemeinen und einen speziellen Teil unterteilt: Im anfänglichen Teil werden die Grundlagen, Prinzipien und Ergebnisse der Fast Track Rehabilitation und die aktuelle Literatur hierzu dargestellt. Dieser Teil ist sehr praxisnah verfasst und bietet sowohl dem jungen als auch dem erfahrenen Arzt einen detaillierten Überblick über die pathophysiologischen und klinischen Hintergründe der Thematik - von der prä- über die intraoperative Phase bis hin zum postoperativen Vorgehen. Der Text ist übersichtlich strukturiert, weist eine Vielzahl von Tabellen auf und wird häufig durch ein farblich abgesetztes „Fazit" der einzelnen Unterpunkte zusammengefasst. In den folgenden 5 Sektionen des speziellen Teils werden exemplarisch konkrete perioperative Behandlungspfade häufiger operativer Eingriffe für die Bereiche der Allgemein- und Viszeralchirurgie (von der Hernien- über die Kolon- und endokrine Chirurgie bis zur hepatopankreatobiliären Chirurgie), der Gefäß-, der Thoraxchirurgie, der Gynäkologie und Urologie aufgeführt.
Die überwiegend tabellarische Darstellung gibt einen ausführlichen Zeitplan und Weg des Patienten in der Klinik vor und behandelt stichwortartig die Abläufe in den verschiedenen Fachbereichen anhand von übersichtlich nebeneinander stehenden Algorithmen. DRG-Daten zu den einzelnen Bereichen runden die relevanten Informationen ab. Das Ziel der Etablierung klinischer Pfade in der eigenen Klinik kann mit dieser Hilfestellung durch Adaptation der Vorlagen deutlich einfacher realisiert werden. Auf der beigefügten $C D$ finden sich zu allen beschriebenen Erkrankungen Texte zur prä- und postoperativen Patienteninformation sowie zur Information für den Hausarzt, die ausgedruckt und an die eigenen Abläufe angepasst werden können.

Zusammenfassend bietet das vorliegende Werk Einsteigern und auch erfahrenen Ärzten einen aktuellen und umfassenden Überblick über das Thema der Fast Track Rehabilitation und eignet sich auch für die Pflegefachberufe. In gut strukturierten, praxisnahen klinischen Pfaden erhält der Leser eine wertvolle Unterstützung für die Etablierung dieser modernen Konzepte an der eigenen Klinik.

R. Kalff(Jena). 